\title{
P300-Based Chinese Speller: A Systematic Summary
}

\author{
Xiang-Qian Liu ${ }^{1, *}$, Min Jiang ${ }^{1, * *}$, Fei Chao ${ }^{1,{ }^{* * *}}$, Chang-Le Zhou ${ }^{1, * * * *}$, Ming-Hui Shi ${ }^{1, * * * * *}$ \\ ${ }^{1}$ Department of Cognitive Science, School of Information Science and Engineering, Xiamen University, Xiamen 361005, China; \\ Fujian Key Laboratory of Brain-inspired Computing Technique and Applications (Xiamen University), Xiamen 361005, China \\ *31520151154115@stu.xmu.edu.cn, ${ }^{\star *}$ minjiang@xmu.edu.cn, ${ }^{* * *}$ fchao@xmu.edu.cn, ${ }^{* * *}$ dozero@xmu.edu.cn, ${ }^{* * * *}$ smh@xmu.edu.cn
}

\begin{abstract}
Evoked by the oddball events, P300 potential is closely related to the advanced psychological activities of human beings, and plays a significant role in the electroencephalogram (EEG)-based brain-computer interface (BCI) systems. Utilizing the principle of eliciting P300, we can implement BCIs to input characters, which enable people to express their intentions directly by brain. In recent years, P300-based English speller (P300ES) has been widely studied while P300-based Chinese speller (P300CS) has been far less fully investigated, owing to the complexity of sinograms. This paper aims at making a systematic summary of the current P300CS systems, and showing that P300CS would be a promising research field deserving of further researches in the furture.
\end{abstract}

\section{Introduction}

The ability to communicate with other people is one of the main factors that makes our lives meaningful. However, patients suffering from amyotrophic lateral sclerosis (ALS) or brainstem damage may lose control over voluntary muscles and require assistive technologies to help them communicate.

Brain-computer interfaces (BCIs) provide a promising means to fulfill basic communication needs for such people. A brain-computer interface is a system which uses brain signals to establish direct communication pathways between human brains and external devices, without the involvement of peripheral nerves and muscles [1].

Depending on the brian signals used, there are many types of BCIs, such as: electroencephalogram (EEG)-based BCI, magnetoencephalogram (MEG)-based BCI, functional magnetic resonance imaging (fMRI)-based $\mathrm{BCI}$, functional near infrared spectroscopy (fNIRS)-based BCI. EEG is the general term for various electrical activities induced by cortical neurons, and it can be sensed through a set of electrodes placed on the scalp of subjects. Due to the superiority of high time resolution and low-cost acquisition devices, EEG has been the most commonly used brain signal in noninvasive BCIs at present [2].

As a well-known component of endogenous EEG, P300 was firstly discovered by Sutton et al in 1965 [3]. It is a positive peak in the EEG signals, appearing approximately
$300 \mathrm{~ms}$ after the presentation of infrequent task-relevant stimuli [4]. To evoke a P300 potential, subjects are required to observe a random sequence of stimuli which the taskrelevant stimulus (oddball event) appears only rarely, while the other stimuli (normal events) appear more often. By determining the occurrence time of P300 response, P300based BCIs can identify which stimulus is task-relevant.

P300-based BCIs have been applied in many aspects, such as recognizing traffic lights [5], dialing phone numbers [6], moving cursors [7], or even controlling a robotic prosthesis [8]. However, the most popular application of P300-based BCIs is spelling [9-12], which enable users to input characters directly by brain. The original P300-based speller was proposed by Farwell and Donchin in 1988 [13]. In their speller, users were presented with a $6 \times 6$ matrix containing 36 characters. The rows and columns of the matrix were flashed in random order. Users were required to concentrate exclusively on the desired character while ignoring others. In this case, P300 could be elicited by the flashing of the row and column containing the desired character, and the desired character was identified as the intersection of the above row and column.

Most of the researches on P300-based spellers are focused on the input of English or other languages written using letters. Few studies have examined the efficacy of spellers for inputting languages that use "strokes", such as Chinese. This is because the number of sinograms is too numerous (more than 11000) for a reasonably sized matrix. Therefore, P300-based Chinese spller (P300CS) is more 
difficult to actualize than P300-based English speller (P300ES).

This article is a systematic summary of the current P300CS systems, the remainder of this paper is organized as follows: Section 2 introduces the implementation methods of P300CS; Section 3 discusses the existing problems and outlines possible lines of future developments of this field; Section 4 concludes the paper.

\section{Implementation Methods of P300CS}

\subsection{Stroke-Based Method}

Sinograms are made up of hierarchical arrangements of strokes, a P300CS could be developed that allow users to input sinograms stroke-by-stroke (stroke-based method).

Employing the stroke-based method, $\mathrm{Wu}$ et al proposed the first P300CS in 2009, using five basic stroke items (I, J, 、, $\rightarrow$ ) and two control items [14]. The items flicked one by one (Fig. 1(a)). When the target item flicked, the P300 response could be evoked in users' EEG signals.

Based on the same method, in 2010, Jin et al developed a more sophisticated P300CS which can send messages on a cell phone [15]. In this speller, a $4 \times 4$ matrix containing five stroke items, eight number items (1-8) and three control items was used to allow sinogram input with P300 (Fig. 1(b)).

However, for traditional Chinese (used in Hongkong and Taiwan), a sinogram may consists of twenty or more strokes, greatly limiting the efficiency of such a P300CS. In 2010, Minett et al presented a novel matrix devoted to speed up selection process of stroke-based method [16]. In the novel matrix, each item represents a subcharacter comprising multiple strokes (Fig. 1(c)), users can make smaller number of selections by inputting sinograms subcharacter-bysubcharacter. The subcharacter-by-subcharacter approach could be seen as an improvement of stroked-based method.

\subsection{Phonetics-Based Method}

Each sinogram has its own pronunciation, P300CS could also be exploited to allow sinogram input with phonetics (phonetics-based method). There are two types of phonetics used in China: Zhuyin (used in Taiwan) and Pinyin (used in maninland). Correspondingly, phonetics-based method can be divided into the Zhuyin-based method and the Pinyinbased method.

In 2011, Sun et al reported the first phonetics-based P300CS adopting Zhuyin [17]. This BCI constructed a $3 \times 4$ matrix containing 11 groups of Zhuyin items and one control item (Fig. 2(a)), a whole row or column of the matrix was intensified at a time. The speller attained acceptable results, and proved the feasibility of phonetics-based method.

Another P300CS based on phonetics (Pinyin) was proposed by $\mathrm{Xu}$ et al in 2015 [18], their work can be seen in Fig. 2(b).
Compared with stroke-based method, phonetics-based method is more in line with people's inputting habits. However, it can't cover up the reality that the same phonetics generally correspond to several different sinograms, which means phonetics-based method might result in many optional sinograms.
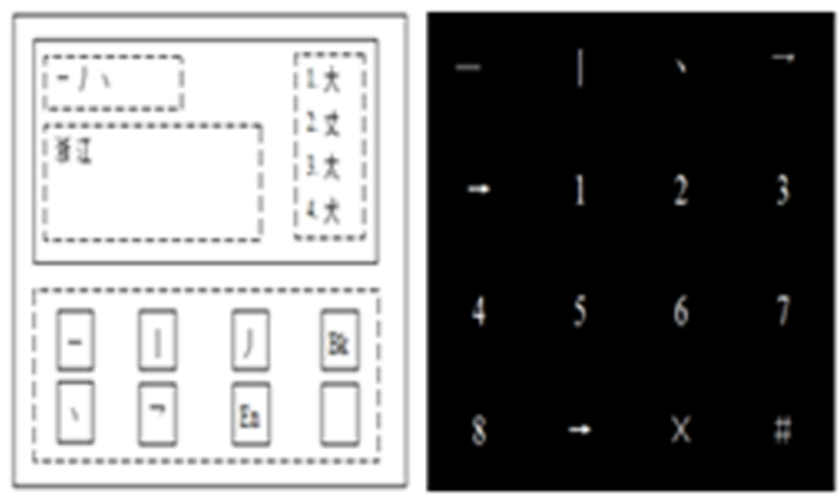

(a) (adapt from [14])

(b) (adapt from [15])

\begin{tabular}{|c|c|c|c|c|}
\hline- & I & J & 1 & 乙 \\
\hline 㜾 & そ & 儿 & 才 & 八 \\
\hline 打 & 口 & $\Gamma$ & 白 & 中 \\
\hline 火 & 言 & 日 & 鉄 & 寺 \\
\hline 們 & 江 & 木 & 天 & 大 \\
\hline- & + & 石 & 戗 & 山 \\
\hline
\end{tabular}

(c) (adapt from [16])

Figure1. Stroke-based method display.

\begin{tabular}{|c|c|c|c|}
\hline $\begin{array}{c}\text { ケタ } \\
\text { Yこむ }\end{array}$ & $\begin{array}{c}\text { Пᄃ } \\
\text { せ采 }\end{array}$ & $\begin{array}{l}\text { 万㕕 } \\
\text { 幺又 }\end{array}$ & $\begin{array}{c}4 \\
-x\end{array}$ \\
\hline $\begin{array}{l}\text { 3⿻ } \\
\text { 日、 }\end{array}$ & $\begin{array}{l}\text { 《 } \\
\text { 乃 }\end{array}$ & $\begin{array}{l}\Gamma \zeta \\
\angle ル\end{array}$ & $\begin{array}{r}<T \\
\mathbf{U}\end{array}$ \\
\hline 世 & そち & $\begin{array}{c}\text { Pム } \\
\vee\end{array}$ & $\longleftarrow$ \\
\hline
\end{tabular}

(a) (adapt from [17])

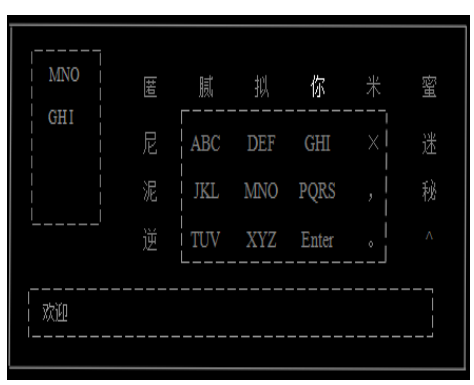




\section{(b) (adapt from [18])}

Figure2. Phonetics-based method display.

\section{Discussion}

P300CS systems are normally characterized by low rates of accuracy and information transfer [19], and there are several problems in the current research.

First, most of the current works on P300CS are intended to prove the feasibility of their solutions, not to provide a practicable system. Then, most of the current approaches employed in P300CS are modeled on P300ES, without considering the uniqueness of sinograms. In addition, the experimens are mostly peroformed only on the healthy students in college, the very limited range of subjects may lead to unrepresentative results.

Regarding the future research of P300CS, the development trends of this research field are discussed in this section too.

First, P300CS should be more user-friendly. P300-based $\mathrm{BCIs}$ are required to present many repetitive stimuli during use, which will inevitably lead to the problem of fatigue. How to improve the comfort degree of system is a issue deserves more research. Then, the non-P300 components could be taken in account. P300-based BCIs typically rely on not only the P300, but also other EEG components such as N100, N200, N400 and P600. For example, Scholars found that the face paradigm (items are transparently overlaid with human faces) could accentuated the N170 and N400 which had a high signal to noise ratio and significantly improved the classification accuracy [20, 21]. Finally, P300CS could be enhanced by adding a statistical language model. Most of the current P300CS treat sinogram selections as an independent process with no prior information. The statistical language model could provide the function of language prediction, which means the subsequent target sinograms are more likely to be selected utilizing the information information of sinograms inputted previously. For instance, a P300-based speller conjunction with a statistical language model was tested in [22], its high performance had showed the efficiency of language prediction function.

\section{Conclusion}

P300CS allows for sinogram input directly by brain. A systematic summary of the current P300CS systems is made in this paper. Firstly, the implementation methods (strokebased method and phonetics-based method) of P300CS is introduced. Then, the existing problems and the future development directions of this field are discussed. Although there have been several works tentative to explore the P300CS, it is not enough. It is sincerely hoped that more in- depth studies can be conducted in this research field in the future.

\section{Acknowledgment}

The project was supported by the Natural Science Foundation of Fujian Province of China (No.2017J01128 and 2017J01129) and by the National Natural Science Foundation of China (No.61673322 and 61673328).

\section{References}

[1] J. R. Wolpaw and D. J. McFarland, "Brain-computer interfaces for communication and control," Clinical Neurophysiology, vol. 113, pp. 767-791, 2002.

[2] E. C. Leuthardt, G. Schalk, J. R. Wolpaw, and J. G. Ojemann, "A brain-computer interface using electrocorticographic signals in humans," Journal of Neural Engineeringy, vol. 1, pp. 63-71, 2004.

[3] S. Sutton, M. Braren, J. Zubin, and E. R. John, "Evoked-potential correlates of stimulus uncertainty," Science, vol. 150, pp. 1187-1188, 1965.

[4] J. Polich, "On the relationship between EEG and P300: Individual differences, aging, and ultradian rhythms," International Journal of Psychophysiology, vol. 26, pp. 299-317, 1997.

[5] J. D. Bayliss and D. H. Ballard, "Single trial P3 epoch recognition in a virtual environment," Neurocomputing, vol. 32, pp. 637-642, 2000.

[6] A. R. Miller, J. P. Rosenfeld, M. Soskins, and M. Jhee, "P300 Amplitude and topography in an autobiographical oddball paradigm involving simulated amnesia," International Journal of Psychophysiology, vol. 16, pp. 1-11, 2002.

[7] J. R. Wolpaw, D. J. McFarland, G. W. Neat, and C. A. Forneris, "An EEG-based brain-computer interface for cursor control," Electroencephalography and clinical neurophysiology, vol. 78, pp. 252-259, 1991.

[8] R. T. Lauer, P. H. Peckham, K. L. Kilgore, and W. J. Heetderks, "Applications of cortical signals to neuroprosthetic control: A critical review," IEEE Transactions on Rehabilitation Engineering, vol. 8, pp. 205-208, 2000.

[9] B. Z. Allison and J. A. Pineda, "ERPs evoked by different matrix sizes: implications for a brain computer interface (BCI) system," IEEE Transactions on Neural Systems and Rehabilitation Engineering, vol. 11, pp. 110-113, 2003.

[10]E. W. Sellers and D. J. McFarland, "Toward enhanced P300 speller performance," Journal of Neuroscience Methods, vol. 167, pp. 15-21, 2008. 
[11]N. Xu, X. Gao, and B. Hong, "BCI Competition 2003Data set IIb: enhancing P300 wave detection using ICA based subspace projections," IEEE Transactions on Bio-medical Engineering, vol. 51, pp. 1067-1072, 2004.

[12]R. Fazel-Rezai and K. Abhari, "A region-based P300 speller for brain-computer interface," Canadian Journal of Electrical and Computer Engineering, vol. 34, pp. 81-85, 2009.

[13]L. A. Farwell and E. Donchin, "Talking off the top of your head: Toward a mental prosthesis utilizing eventrelated brain potentials," Electroencephalography and clinical neurophysiology, vol. 70, pp. 510-523, 1988.

[14]B. Wu, Y. Su, J. Zhang, and X. Li, "A Virtual Chinese Keyboard BCI System Based on P300 Potentials," Acta Electronica Sinica, vol. 37, pp. 1733-1738, 2009.

[15] J. Jin, B. Z. Allison, C. Brunner, and B. Wang, "P300 Chinese input system based on Bayesian LDA," Biomedizinische Technik Biomedical Engineering, vol. 55, pp. 5-18, 2010.

[16] J. Minett, G. Peng, L. Zhou, H. Y. Zheng, and S. Wang, "An Assistive Communication Brain-Computer Interface for Chinese Text Input," 4th International Conference on Bioinformatics and Biomedical Engineering, Jun. 2010.
[17]K. T. Sun, T. W. Huang, and M. C. Chen, "Design of Chinese Spelling System Based on ERP," 11th International Conference on Bioinformatics and Bioengineering, vol. 1997, pp. 310-313, Jun. 2010.

[18]X. Xu and H. J. Fang, "A P300-based BCI system for Online Chinese input," Journal of Huaqiao University (Natural Science), vol. 36, pp. 269-274, 2015.

[19] T. W. Huang, Y. H. Tai, Y. J. Tian, and K. T. Sun, "The fastest BCI for writing Chinese characters using brain waves," 4th Global Congress on Intelligent Systems, pp. 346-349, 2013.

[20]T. Kaufmann, S. M. Schulz, C. Grünzinger, and A. Kübler, "Flashing characters with famous faces improves ERP-based brain-computer interface performance," Journal of Neural Engineering, vol. 8, pp. 173-178, 2011.

[21] Y. Zhang, Q. Zhao, J. JIn, and X. Wang, “A novel BCI based on ERP components sensitive to configural processing of human faces," Journal of Neural Engineering, vol. 9, pp. 26018-26030, 2012.

[22]D. B. Ryan, G. E. Frye, G. Townsend, D. R. Berry, and S. Mesag, "Predictive spelling with a P300-based braincomputer interface: Increasing the rate of communication," International journal of humancomputer interaction, vol. 27, pp. 69-84, 2011. 